\title{
Editorial: Current Trends in Image Processing and Pattern Recognition
}

\author{
KC Santosh ${ }^{* t}$ \\ PAMI Research Lab, Computer Science, University of South Dakota, Vermillion, SD, United States
}

Keywords: artificial intelligence, computer vision, machine learning, image processing, signal processing, pattern recocgnition

Editorial on the Research Topic

Current Trends in Image Processing and Pattern Recognition

Technological advancements in computing multiple opportunities in a wide variety of fields that range from document analysis (Santosh, 2018), biomedical and healthcare informatics (Santosh et al., 2019; Santosh et al., 2021; Santosh and Gaur, 2021; Santosh and Joshi, 2021), and biometrics to intelligent language processing. These applications primarily leverage AI tools and/or techniques, where topics such as image processing, signal and pattern recognition, machine learning and computer vision are considered.

With this theme, we opened a call for papers on Current Trends in Image Processing \& Pattern Recognition that exactly followed third International Conference on Recent Trends in Image Processing \& Pattern Recognition (RTIP2R), 2020 (URL: http://rtip2r-conference.org). Our call was not limited to RTIP2R 2020, it was open to all. Altogether, 12 papers were submitted and seven of them were accepted for publication.

In Deshpande et al., authors addressed the use of global fingerprint features (e.g., ridge flow,

Edited and reviewed by: Antonio Fernández-Caballero, University of Castilla-La Mancha,

Spain

*Correspondence: KC Santosh santosh.kc@ieee.org

tORCID:

KC Santosh

orcid.org/0000-0003-4176-0236

Specialty section: This article was submitted to Human and Medical Genomics, a section of the journal

Frontiers in Genetics

Received: 28 September 2021

Accepted: 06 October 2021

Published: 09 December 2021

Citation:

Santosh KC (2021) Editorial: Current Trends in Image Processing and

Pattern Recognition.

Front. Robot. Al 8:785075.

doi: 10.3389/frobt.2021.785075 frequency, and other interest/key points) for matching. With Convolution Neural Network (CNN) matching model, which they called "Combination of Nearest-Neighbor Arrangement Indexing (CNNAI)," on datasets: FVC2004 and NIST SD27, their highest rank-I identification rate of $84.5 \%$ was achieved. Authors claimed that their results can be compared with the stateof-the-art algorithms and their approach was robust to rotation and scale. Similarly, in Deshpande et al., using the exact same datasets, exact same set of authors addressed the importance of minutiae extraction and matching by taking into low quality latent fingerprint images. Their minutiae extraction technique showed remarkable improvement in their results. As claimed by the authors, their results were comparable to state-of-the-art systems.

In Gornale et al., authors extracted distinguishing features that were geometrically distorted or transformed by taking Hu's Invariant Moments into account. With this, authors focused on early detection and gradation of Knee Osteoarthritis, and they claimed that their results were validated by ortho surgeons and rheumatologists.

In Tamilmathi and Chithra, authors introduced a new deep learned quantization-based coding for $3 \mathrm{D}$ airborne LiDAR point cloud image. In their experimental results, authors showed that their model compressed an image into constant 16-bits of data and decompressed with approximately $160 \mathrm{~dB}$ of PSNR value, $174.46 \mathrm{~s}$ execution time with $0.6 \mathrm{~s}$ execution speed per instruction. Authors claimed that their method can be compared with previous algorithms/techniques in case we consider the following factors: space and time.

In Tamilmathi and Chithra, authors carefully inspected possible signs of plant leaf diseases. They employed the concept of feature learning and observed the correlation and/or similarity between symptoms that are related to diseases, so their disease identification is possible. 
In Das Chagas Silva Araujo et al, authors proposed a benchmark environment to compare multiple algorithms when one needs to deal with depth reconstruction from two-event based sensors. In their evaluation, a stereo matching algorithm was implemented, and multiple experiments were done with multiple camera settings as well as parameters. Authors claimed that this work could be considered as a benchmark when we consider robust evaluation of the multitude of new techniques under the scope of event-based stereo vision.

In Steffen et al.; Gornale et al., authors employed handwritten signature to better understand the behavioral biometric trait for

\section{REFERENCES}

Santosh, KC, Antani, S., Guru, D. S., and Dey, N. (2019). Medical Imaging Artificial Intelligence, Image Recognition, and Machine Learning Techniques. United States: CRC Press. ISBN: 9780429029417. doi:10.1201/ 9780429029417

Santosh, KC, Das, N., and Ghosh, S. (2021). Deep Learning Models for Medical Imaging, Primers in Biomedical Imaging Devices and Systems. United States: Elsevier. eBook ISBN: 9780128236505.

Santosh, KC (2018). Document Image Analysis - Current Trends and Challenges in Graphics Recognition. United States: Springer. ISBN 978-981-13-2338-6. doi:10.1007/978-981-13-2339-3

Santosh, KC, and Gaur, L. (2021). Artificial Intelligence and Machine Learning in Public Healthcare: Opportunities and Societal Impact. Spain: SpringerBriefs in Computational Intelligence Series. ISBN: 978-981-16-6768-8. doi:10.1007/978981-16-6768-8

Santosh, KC, and Joshi, A. (2021). COVID-19: Prediction, Decision-Making, and its Impacts, Book Series in Lecture Notes on Data Engineering and Communications document authentication/verification, such letters, contracts, and wills. They used handcrafter features such as LBP and HOG to extract features from 4,790 signatures so shallow learning can efficiently be applied. Using k-NN, decision tree and support vector machine classifiers, they reported promising performance.

\section{AUTHOR CONTRIBUTIONS}

The author confirms being the sole contributor of this work and has approved it for publication.

Technologies. United States: Springer Nature. ISBN: 978-981-15-9682-7. doi:10.1007/978-981-15-9682-7

Conflict of Interest: The author declares that the research was conducted in the absence of any commercial or financial relationships that could be construed as a potential conflict of interest.

Publisher's Note: All claims expressed in this article are solely those of the authors and do not necessarily represent those of their affiliated organizations, or those of the publisher, the editors and the reviewers. Any product that may be evaluated in this article, or claim that may be made by its manufacturer, is not guaranteed or endorsed by the publisher.

Copyright (c) 2021 Santosh. This is an open-access article distributed under the terms of the Creative Commons Attribution License (CC BY). The use, distribution or reproduction in other forums is permitted, provided the original author(s) and the copyright owner(s) are credited and that the original publication in this journal is cited, in accordance with accepted academic practice. No use, distribution or reproduction is permitted which does not comply with these terms. 